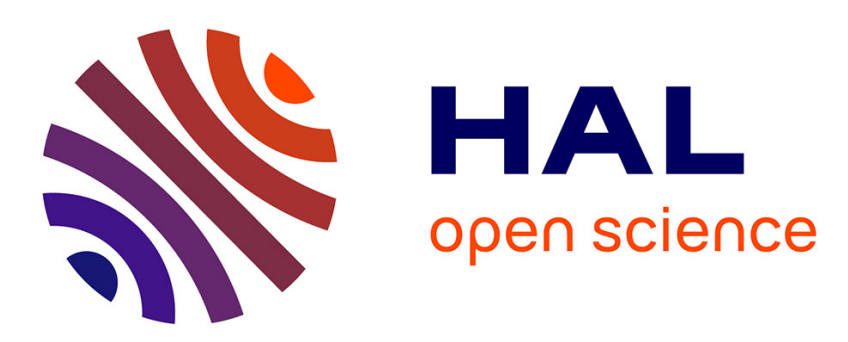

\title{
Woodwind instrument design optimization based on impedance characteristics with geometric constraints
}

Augustin Ernoult, Christophe Vergez, Samy Missoum, Philippe Guillemain, Michael Jousserand

\section{- To cite this version:}

Augustin Ernoult, Christophe Vergez, Samy Missoum, Philippe Guillemain, Michael Jousserand. Woodwind instrument design optimization based on impedance characteristics with geometric constraints. Journal of the Acoustical Society of America, 2020, 148 (5), pp.2864-2877. $10.1121 / 10.0002449$. hal-02479433v3

\section{HAL Id: hal-02479433 \\ https://hal.science/hal-02479433v3}

Submitted on 30 Nov 2020

HAL is a multi-disciplinary open access archive for the deposit and dissemination of scientific research documents, whether they are published or not. The documents may come from teaching and research institutions in France or abroad, or from public or private research centers.
L'archive ouverte pluridisciplinaire HAL, est destinée au dépôt et à la diffusion de documents scientifiques de niveau recherche, publiés ou non, émanant des établissements d'enseignement et de recherche français ou étrangers, des laboratoires publics ou privés. 


\title{
Woodwind instrument design optimization based on impedance characteristics with geometric constraints
}

\author{
Augustin Ernoult ${ }^{(1) *}$, Christophe Vergez ${ }^{(2)}$, Samy Missoum ${ }^{(3)}$, \\ Philippe Guillemain ${ }^{(2)}$, Michael Jousserand ${ }^{(4)}$
}

1. Magique 3D team, Inria Bordeaux Sud Ouest, 200 avenue de la vieille tour, 33405 Talence Cedex, France

2. Aix Marseille Univ., CNRS, Centrale Marseille, LMA UMR 7031, Marseille, France

3. Aerospace and Mechanical Engineering Department, University of Arizona, Tucson, Arizona, 85721, USA

4. Buffet Crampon, 5 rue Maurice Berteaux, 78711 Mantes-La-Ville, France

\begin{abstract}
Summary
Computational optimization algorithms coupled with acoustic models of wind instruments provide instrument makers with an opportunity to explore new designs. Specifically, they enable the automatic discovery of geometries exhibiting desired resonance characteristics. In this paper, the design optimization of woodwind instruments with complex geometrical features (e.g., non-cylindrical bore profile and side holes with various radii and chimney heights) is investigated. Optimal geometric designs are searched so that their acoustic input impedance has peaks with specific target frequencies and amplitudes. However, woodwind instruments exhibit complex input impedance whose features, such as resonances, might have non-smooth evolution with respect to design variables, thus hampering gradient-based optimization. For this reason, this paper introduces new formulations of the impedance characteristics (resonance frequencies and amplitudes) using a regularized unwrapped angle of the reflection function. The approach is applied to an illustrative instrument subjected to geometric constraints similar to the ones encountered by manufacturers (a keyless pentatonic clarinet with two-registers). Three optimization problems are considered, demonstrating a strategy to simultaneously adjust several impedance characteristics on all fingerings.
\end{abstract}

\section{Introduction}

Wind instrument makers typically rely on their empirical knowledge to improve an existing instrument or to design a new model. For instance, their experience enables them to select suitable inner geometries of the instrument. Once a new prototype is manufactured, maybe with the help of bespoke tools (e.g., reamers, mandrels), musicians can then evaluate the quality of the instrument. Modifications can be implemented based on the manufacturer's experience to mitigate problems that might have arisen. This procedure is repeated until a satisfactory instrument, which is typically a compromise between different desired features (e.g., tuning of all notes), is reached. This iterative process, which often requires building many prototypes, is costly and time consuming. In addition, the empirical design and manufacturing processes lead to incremental changes of instruments. For this reason, it is important to provide manufacturers and the musical acoustics community with strategies to facilitate and expand design possibilities of new wind instruments.

From a physical point of view, a wind instrument can be described as a nonlinear sound source coupled with a resonator. The resonator is generally reduced to its linear response represented by its input impedance. It is defined in the frequency domain as the ratio between the acoustic pressure and the volume flow at the entrance of the instrument. The knowledge of the input impedance and more particularly of its modal characteristics (frequencies, peak amplitudes and quality-factors) allows one to explain and predict a large range of behaviors exhibited by wind instruments [1]. Many well established solutions are available to compute the input impedance from the geometry of the resonator with a good accuracy for the majority of resonator geometries $[1,2,3,4,5$, $6,7,8]$. The input impedance may therefore be useful to obtain resonance frequencies and peak amplitudes of a given instrument without the need to build a physical prototype $[9,10]$. If the target (desired) characteristics of the input impedance are known, it is also possible to use optimization techniques to solve the inverse problem and iteratively converge towards an optimal resonator geometry with desired impedance characteristics. The

*augustin.ernoult@inria.fr 
ability of optimization algorithms to find solution in a large design space can lead to unexpected configurations, sometimes markedly different from existing instruments.

However, the design optimization of wind instruments can be hampered by several challenges. Due to their nonlinear nature, wind instruments can exhibit drastic changes in behaviors for small variations of their design parameters (e.g., leading to different regimes of oscillation). This high sensitivity of the acoustic behavior can be accounted for through a complete modeling of the instrument and digital sound synthesis. Another difficulty stems from the dimensionality of the optimization problem. For instance, in Tournemenne et al. [11], an optimization was carried out based on a target playing frequency. The optimization algorithm, which was tailored to tackle the complex dynamic behavior of the self-oscillating system, was used with 10 variables at most. However, considering complex bore and holes geometries would require many more design variables, which is the main reason why most authors work on optimization methods based on the input impedance only. Kausel [12] ran an optimization with 100 variables on a brass instrument using a zero-order optimization approach. Gradient-based optimization methods with analytical gradients were also used (Noreland [13]) for the optimization of conical pipes with a up to 400 variables defining the brass bore profile. Strategies to reduce the number of design variables can also be found in the literature. For instance, Braden [14] used a parameterization of the geometry of a brass bell through a few Bessel horns. The choice of the impedance characteristics to optimize is also crucial in the optimization process. When the entire impedance is considered, such as for the bore reconstruction of brass instruments [12] or to approximate a real saxophone by a bicylindrical resonator [15], a norm of the difference to the target impedance over an entire frequency band is generally used. For the design of brass instruments, cost functions based on the tuning of the resonance frequencies are convenient $[13,14]$. This approach alone is not satisfactory for the optimization of woodwind instruments [16]. To design their register hole, the magnitude of the impedance peaks must also be considered, as proposed by Guilloteau [17]. It is therefore necessary to simultaneously adjust different types of input impedance characteristics.

This article deals with the optimization of some geometrical features of a woodwind instrument, including the location and dimensions of the holes. Different cost functions and constraints are considered, relying exclusively on the modal characteristics of the input impedance for all fingerings. The instrument can be seen as a lattice of branched tubes (side holes). As a consequence, secondary resonances, cutoff frequencies due to the holes $[18,19]$, and input impedances of increased complexity can occur. Resonances can appear, disappear or cross another resonance as a result of a small geometric change. Therefore, a gradient-based optimization based on a traditional resonance tracking procedure, with each resonance identified by its order of appearance, would be hindered by the potential non-smoothness of the response with respect to the design variables. This difficulty had not been identified in previous papers on woodwind instruments design optimization [16, 20]. This may be due to the early focus on the so-called logical clarinets whose regular geometries limit the complexity of their input impedance, except for high frequencies. To address the numerical difficulties when dealing with the impedance of instruments with complex geometries, a new differentiable phase-based description of the resonances is used to drive the optimization.

The input impedance model is presented in Section 2. The proposed new differentiable phase-based formulation to characterize resonances is explained in Section 3. Section 4 presents the general optimization problem formulation and the algorithm used. Finally, in Section 5, the proposed approach is demonstrated on the design optimization of an illustrative key-less pentatonic clarinet with two-registers. Three optimization problems are considered involving target resonance frequencies or peak amplitude ratios as well as geometric constraints. A reference configuration is obtained through a low frequency approximation. The influence of the initial design and the robustness of the algorithm are discussed in Section 6

\section{Input impedance model of a woodwind instrument}

Consider a generic woodwind instrument as depicted in Figure 1. The instrument consists of an assembly of $N_{\text {elem }}$ cylindrical or conical elements with or without side holes. The $n^{\text {th }}$ element is characterized using four geometric parameters (Fig. 1): the distance to the previous element $l(n)$, the main pipe radius $r_{\text {pipe }}(n)$ and, if there is a hole, the radius $r_{\text {hole }}(n)$ and the chimney height $h_{\text {hole }}(n)$. Note that in real-world instruments, these geometric properties must satisfy specific constraints, which is a primal aspect of this research.

To characterize the acoustical properties and optimize the design of the instrument, the input impedance $Z$ of its resonator is used:

$$
Z=\frac{A_{t o t} Z_{r a d}+B_{t o t}}{C_{t o t} Z_{r a d}+D_{t o t}},
$$

where $Z_{\text {rad }}$ is the radiation impedance at the output of the resonator. $A_{t o t}, B_{t o t}, C_{t o t}, D_{t o t}$ are the coefficients of the transfer matrix $M_{t o t}$ between the input and the output of the resonator. This total transfer matrix is 


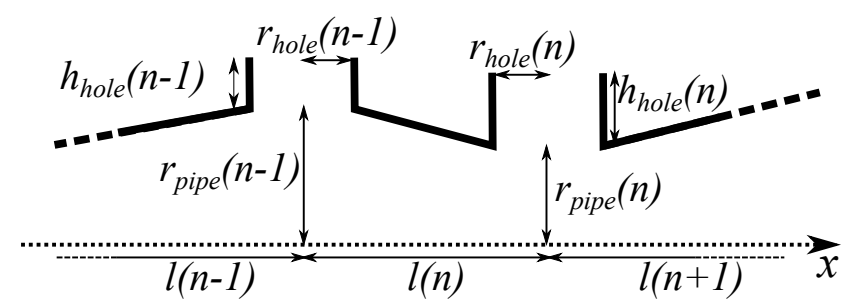

Figure 1: Sketch of the resonator of a generic woodwind instrument defined as a chain of basic elements (e.g., conical/cylindrical segments and holes)

computed by the product of $N_{\text {elem }}$ elementary transfer matrices $M_{i}[1]$ :

$$
M_{\text {tot }}=\prod_{i=1}^{N_{\text {elem }}} M_{i}
$$

Such a transfer matrix may describe a conical or cylindrical pipe or a side hole, open or closed. The conical pipes (convergent, divergent or cylinders) are modeled based on the formulation which accounts for thermoviscous losses proposed by Hélie [6]. The conical element transfer matrix is:

$$
M_{\text {cone }}=\left(\begin{array}{ll}
A_{\text {cone }} & B_{\text {cone }} \\
C_{\text {cone }} & D_{\text {cone }}
\end{array}\right),
$$

where

$$
\left\{\begin{array}{l}
A_{\text {cone }}=\frac{r_{\max }}{r_{\min }} \cosh (\Gamma L)-\frac{\left(r_{\max }-r_{\min }\right)}{r_{\min } \Gamma L} \sinh (\Gamma L) \\
B_{\text {cone }}=\frac{\sin }{\pi r_{\min } r_{\max }} \sinh (\Gamma L) \\
C_{\text {cone }}=\left(A_{\text {cone }} D_{\text {cone }}-1\right) / B_{\text {cone }} \\
D_{\text {cone }}=\frac{r_{\max }}{r_{\min }} \cosh (\Gamma L)+\frac{\left(r_{\max }-r_{\min }\right)}{r_{\max } \Gamma L} \sinh (\Gamma L)
\end{array}\right.
$$

where $r_{\min }$ and $r_{\max }$ are respectively the smallest and biggest radii of the cone, $L$, the curvilinear length (wall length), $\rho$ and $c$ are the air density and the sound velocity respectively. The complex wavenumber $\Gamma$, which accounts for thermoviscous losses, is defined as:

$$
\left\{\begin{array}{l}
\Gamma^{2}=(j k)^{2}+2 \varepsilon k^{3 / 2} \\
\varepsilon=\varepsilon^{*} \sqrt{1-\left(\frac{r_{\max }-r_{\min }}{L}\right)^{2}} \frac{\log \left(1+\frac{r_{\max }-r_{\min }}{r_{\min }}\right)}{r_{\max }-r_{\min }},
\end{array}\right.
$$

$k=\omega / c$ is the wavenumber and $\varepsilon^{*}$ is a coefficient related to the temperature [6]. Note that the ratio in the form $\log (1+x) / x$ has a finite limit at $x=0$ (i.e., $r_{\max }=r_{\min }$ ) which might not be accurately estimated numerically. It must be treated with special attention for elements transitioning from a cone to a cylinder or conversely.

The hole transfer matrices are based on a model proposed by Lefebvre et al. [21]

$$
M_{\text {hole }}=\left(\begin{array}{cc}
1+\frac{Z_{a}}{2 Z_{s}} & Z_{a}\left(1+\frac{Z_{a}}{4 Z_{s}}\right) \\
\frac{1}{Z_{s}} & 1+\frac{Z_{a}}{2 Z_{s}}
\end{array}\right),
$$

where $Z_{s}$ and $Z_{a}$ are two impedances, which have different expressions for open and closed holes. They account for the presence of chimneys and include various length corrections due to the T-joint between the main bore pipe and the chimney hole pipe [21]. Thermoviscous losses in the chimneys are also considered. They influence the amplitude of the resonances, especially for a long and thin chimney, which is generally the case for the register hole. It is noteworthy that the model proposed by Lefebvre et al. [21] accounts for a frequency-dependent internal length correction. However this dependence is neglected as it does not significantly affect the lower modes, which are the focus of this article, while markedly increasing the computational time.

Radiation impedance modeling depends on various assumptions and can be quite difficult especially when side holes are present. The holes can be either directly drilled in the main pipe or extended through chimneys, with or without key pads [22]. In this study, the instrument is key-less with thin-walled side hole chimneys. For simplicity, the radiation impedance of both the main pipe and the side holes are modeled by the unflanged pipe radiation impedance approximated by a non-causal formulation (Silva et al. [23]):

$$
Z_{\text {rad }}=-j \tan \left(k \mathscr{L}_{\text {rad }}(k, r)-j \frac{1}{2} \ln \left|\mathscr{R}_{\text {rad }}(k, r)\right|\right),
$$


where $k$ is the wave number and $\mathscr{L}_{\text {rad }}(k, r)$ and $\mathscr{R}_{\text {rad }}(k, r)$ are rational fractions depending on $k$ and the radius $r$ of the radiating opening. The proposed simplified impedance model is sufficient for the purpose of this design optimization study.

\section{Resonance characteristics}

This article aims to optimize a woodwind instrument based on the peak frequencies and amplitudes of its input impedance. Specifically, resonance frequencies should match specific target values. As for the peak amplitudes, the proposed optimization enforces certain amplitude ratios between peaks. These requirements are targeted through a least-squares formulation based on residuals depending on resonance frequencies and peak amplitudes. The residuals are constructed to ensure their continuity and smoothness in the design domain. This is an essential aspect of this work.

\subsection{Resonance frequencies}

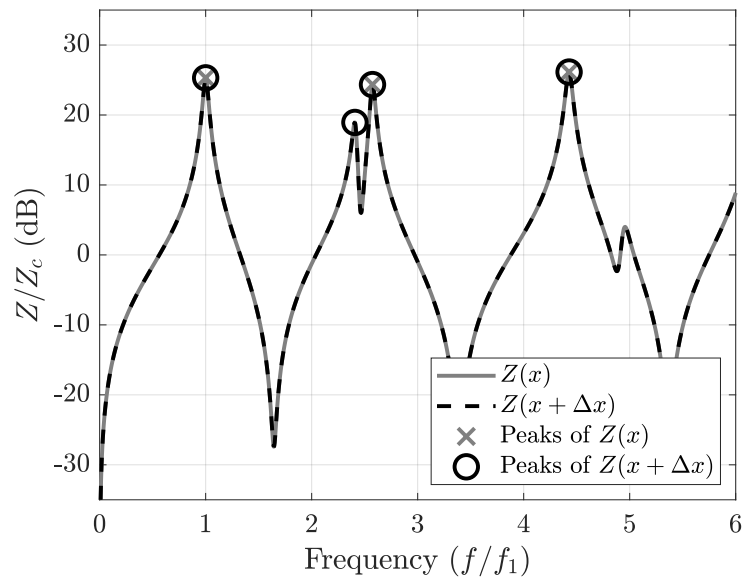

Figure 2: Example of peak detection issue (minimal prominence: $12 \mathrm{~dB}$ ): despite the fact that the two curves are almost superimposed, a supplementary peak is detected at $f \approx 2.3 f_{1}$ for a small variation of the geometry (one design parameter is increased by $\Delta x_{i}=10^{-10}$ and the others are unchanged).

Resonances in woodwind instruments can be defined in various ways such as through the peaks of the impedance modulus [16], the zeros of the imaginary part of the impedance (equivalent to the zeros of its phase), or the real part of its complex poles [24]. Note that these definitions are not equivalent and their relation to the sounding frequency necessitates a detailed description of the sounding mechanism, which is beyond the scope of this study. Here, the sounding mechanism is excluded which gives some freedom on the resonance definition. One must only taking care to chose the corresponding target values consistency with the resonances' definition.

However, woodwind instrument toneholes, which can be seen as multiple parallel tubes, impose local and global cutoff frequencies $[18,19]$. As a consequence, resonance tracking can be problematic, whatever definition of resonance is selected. A small variation of the geometry can lead to the appearance or disappearance of impedance peaks. The frequency of the $m^{t h}$ resonance can therefore jump from one value to another for a small variation of the geometric parameters. Figure 2 provides an example of such a problem for the second resonance. Another problem appears when two peaks cross each other. In that case the derivative of a given order resonance frequency with respect to a design variable suddenly changes sign. In the context of optimization, traditional cost functions based on the tracking of ordered resonances can lead to non-smooth problems. Discontinuities appear for peak detection approaches based the search of the zero crossing of the imaginary part of the impedance, of the derivative of the modulus or of complex poles.

In order to mitigate the consequences of the non-smoothness problems in traditional peak detection techniques, a new approach is proposed which does not involve the explicit enumeration of the peaks to estimate resonance 
a)

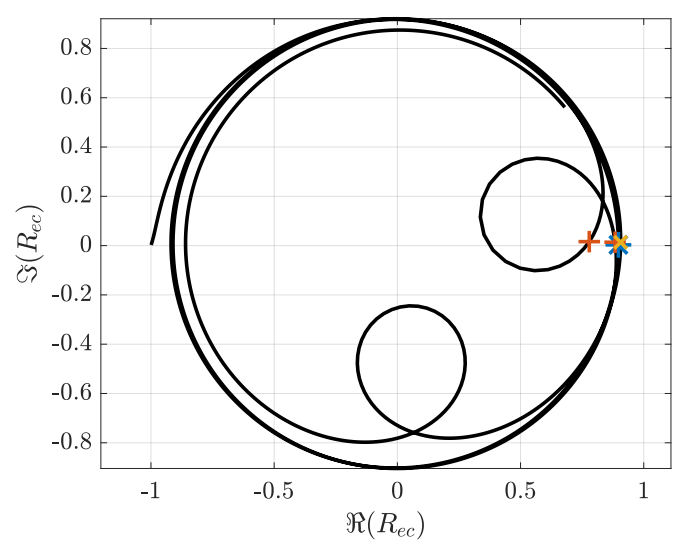

b)

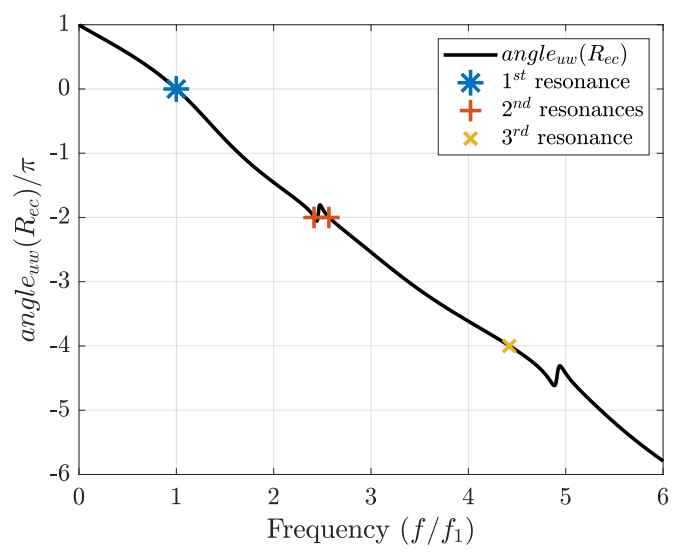

Figure 3: The reflection function $R_{e c}(a)$ corresponding to the impedance of Fig. 2 and its unwrapped phase (b) with the first resonances (markers).

frequencies. Instead, resonances are identified based on the unwrapped phase angle of the reflection function:

$$
R_{e c}=\frac{Z_{i n}-Z_{c}}{Z_{i n}+Z_{c}},
$$

where $Z_{c}=\rho c /\left(\pi r_{\text {pipe }}(1)^{2}\right)$ is the characteristic impedance at the input of the instrument. This reflection function can be seen as the transfer function between incident and reflected waves observed at the input of the resonator. If the resonator induces a frequency-independent time delay between incident and reflected waves (e.g., a cylinder with perfect radiation condition), the phase angle of the reflection function decreases linearly with the frequency. In a real-world resonator, the time delay can be slightly dependent on the frequency but, overall, the phase angle of the reflection function exhibits a decreasing global trend. Furthermore, if resonances are defined as the zeros of the input impedance phase (and decreasing phase to exclude anti-resonances), the phase angle at the resonance frequencies is a multiple of $2 \pi$ (Fig. 3.b). The frequency $f_{m}$ of the $m^{\text {th }}$ resonance is therefore identified as follows:

$$
\operatorname{angle}_{u w}\left(R_{e c}\left(f_{m}\right)\right)=-2 \pi(m-1),
$$

with angle ${ }_{u w}\left(R_{e c}\right)$ the unwrapped angle of the reflection function. This definition enables the identification of the $m^{\text {th }}$ resonance without enumerating the lower ones.

In some cases, due to the cutoff frequency, double resonance frequencies can appear. Figure 2 shows the case of a double second resonance: the condition angle ${ }_{u w}\left(R_{e c}\left(f_{m}\right)\right)=-2 \pi$ is fulfilled for two frequencies (Fig. 3.b). These double resonances are associated with a reflection function which crosses the real axis twice in the complex plane as it forms a loop (Fig. 3.a). A double resonance also leads to variations in the decrease of the phase angle (Fig. 3.b) and leads to problems if the aim is to determine a unique second resonance frequency.

It is typically the case if the cost function of the optimization problem uses the residual between the estimated resonance frequency $f_{m}$ and its target $f_{m}^{\odot}[16]:\left(f_{m}-f_{m}^{\odot}\right)$. However, in this study, this difficulty is avoided by using the resonance definition in Eq. 9, which is only based on the phase at the target resonance frequency. For the purpose of optimization (see Sec. 4.1), the various cost functions will be based on the difference between the unwrapped phase of the reflection function at these frequencies and their target value: $\operatorname{angle}_{u w}\left(R_{e c}\left(f_{m}^{\odot}\right)\right)+2 \pi(m-1)$.

\subsubsection{Computation of the unwrapped angle and regularization}

Based on its definition, the reflection function is differentiable if $Z_{i n}$ is differentiable. However, there are also several sources of discontinuity which might hamper the use of gradient-based optimization. One source of discontinuities stems from the phase definition modulo $2 \pi$. This discontinuity is removed by integration of the phase defined as $\varphi=\arctan \left(\Im\left(R_{e c}\right) / \Re\left(R_{e c}\right)\right)$. The phase derivative with respect to the frequency is:

$$
\frac{d \varphi}{d f}=\frac{1}{\left|R_{e c}\right|^{2}}\left(\Re\left(R_{e c}\right) \frac{d \Im\left(R_{e c}\right)}{d f}-\Im\left(R_{e c}\right) \frac{d \Re\left(R_{e c}\right)}{d f}\right) .
$$


Finally, the unwrapped angle is obtained by integration between the frequencies 0 and $f$ :

$$
\begin{aligned}
\operatorname{angle}_{u w}\left(R_{e c}(f)\right) & =\int_{0}^{f} \frac{\Re\left(R_{e c}\right)}{\left|R_{e c}\right|^{2}} \frac{d \Im\left(R_{e c}\right)}{d f} d f-\int_{0}^{f} \frac{\Im\left(R_{e c}\right)}{\left|R_{e c}\right|^{2}} \frac{d \Re\left(R_{e c}\right)}{d f} d f \\
& =\int_{0}^{f} \cos \varphi \frac{d(\sin (\varphi))}{d f} d f-\int_{0}^{f} \sin (\varphi) \frac{d(\cos (\varphi))}{d f} d f .
\end{aligned}
$$

By construction, this expression removes the discontinuities stemming from the modulo $2 \pi$ definition of $\varphi$.

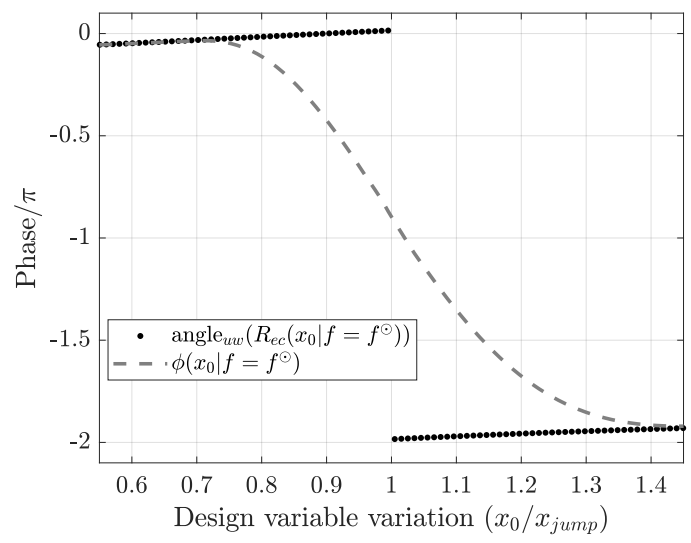

Figure 4: Example of discontinuous unwrapped phase angle at a given frequency $f^{\odot}$ (black dots, computed with Eq.(10)) with respect to a design variable $x_{0}$. The discontinuity occurs at $x_{0}=x_{j u m p}$. The regularized unwrapped angle discussed further (dashed grey line, computed with Eq.(12)).

However, another form of discontinuity may occur, in the context of optimization, as a phase jump of $\pm 2 \pi$ for a small variation of the design variables or other parameters (Figure 4). The phase discontinuity can be interpreted by studying the reflection function in the complex plane. The observed discontinuities occur when the origin of the complex plane "crosses" the representations of the reflection functions obtained for two infinitesimally close designs. Figure 5 provides graphical depiction of this crossing with the corresponding phase discontinuities.

a)
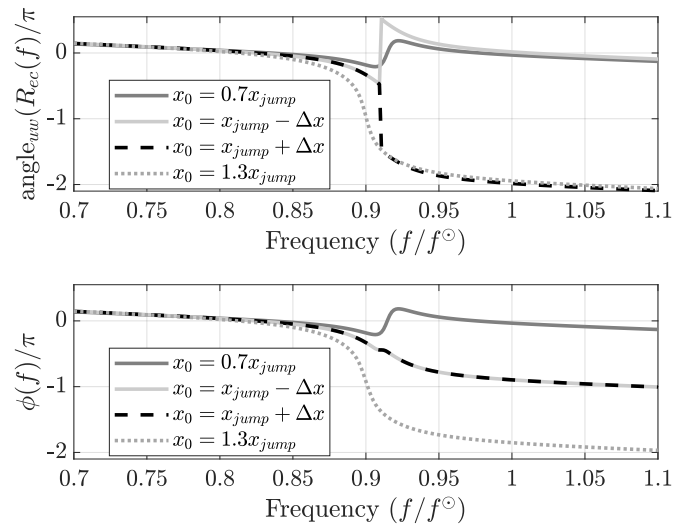

b)

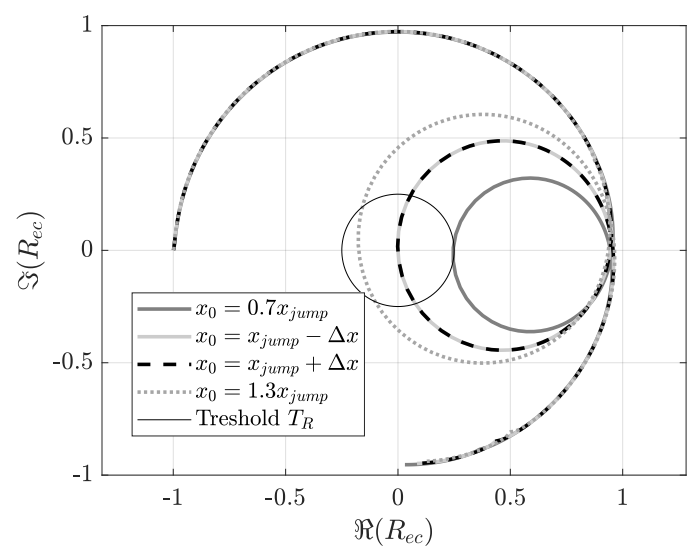

Figure 5: (Left) Unwrapped phase angles for designs leading to discontinuities $\left(\Delta x_{0} / x_{j u m p}=10^{-5}\right)$ and designs away from $x_{\text {jump }}$ : Unwrapped angle computed with Eq.(10) (top), regularized unwrapped angle with removed discontinuity (Eq.(12))(bottom). (Right) Corresponding reflection functions: as the design changes, the phase discontinuity appears when the origin of the plane crosses the reflections functions. The limit of the area where the regularization operates $\left(\left|R_{e c}\right|<T_{R}\right.$, Eq.(11)) is plotted as a black circle for $T_{R}=0.25$.

The discontinuity problem is addressed by regularizing the phase computation in Eq.(10). This is done by introducing a coefficient $\tilde{R}$ in the unwrapped angle integral, which eliminates singularities. The coefficient is 
defined as:

$$
\left\{\begin{array}{l}
\tilde{R}=0.5+0.5 \cos \left(\pi \frac{\left|R_{e c}\right|-T_{R}}{T_{R}}\right) \text { if }\left|R_{e c}\right| \leq T_{R}, \\
\tilde{R}=1 \\
\text { otherwise, }
\end{array}\right.
$$

where $T_{R}$ is an arbitrary threshold. This coefficient is proportional to $\left|R_{e c}\right|^{2}$ when $\left|R_{e c}\right|$ tends towards zero. The regularized unwrapped angle $\phi$ is therefore computed as

$$
\begin{aligned}
\phi(f) & =\int_{0}^{f} \frac{\tilde{R}}{\left|R_{e c}\right|^{2}} \Re\left(R_{e c}\right) \frac{d \Im\left(R_{e c}\right)}{d f} d f-\int_{0}^{f} \frac{\tilde{R}}{\left|R_{e c}\right|^{2}} \Im\left(R_{e c}\right) \frac{d \Re\left(R_{e c}\right)}{d f} d f, \\
& =\int_{0}^{f} \tilde{R} \cos (\varphi) \frac{d(\sin (\varphi))}{d f} d f-\int_{0}^{f} \tilde{R} \sin (\varphi) \frac{d(\cos (\varphi))}{d f} d f .
\end{aligned}
$$

When the regularization is effective $(\tilde{R} \neq 1)$, the unwrapped phase angle error might be relatively large for high frequencies $\left(f>0.91 f^{\odot}\right.$ in Fig. 5, a). This can be a problem for the proposed design optimization. For this reason, a small value of the threshold $T_{R}$ is selected to minimize the phase error, but large enough for the regularization. Based on several experiments, $T_{R}=0.25$ is selected.

\subsection{Resonance amplitudes}

To find the amplitude of the $m^{\text {th }}$ resonance, it is first identified using the phase $\phi$ of the reflection function. In the case where the considered impedance maximum does not exactly correspond to a zero phase, the resonance peak is assumed to belong to the frequency domain $\mathscr{A}_{m}$ defined as

$$
\mathscr{A}_{m}=\left\{f\left|\phi_{0} \geq\right| \phi(f)+2 \pi(m-1) \mid\right\},
$$

with $\phi_{0} \leq \pi$. Because $\mathscr{A}_{m}$ is based on $\phi(f)$, which is not necessarily monotonic (see Sec. 3.1), it can therefore be made of several distinct frequency ranges.

The magnitude of the $m^{\text {th }}$ peak is the maximum of the impedance modulus within this interval. However, the search of maximum is not an operator differentiable with respect to the design variables. To make it differentiable, the maximum is approximated by a discrete approximation of the $L^{p}$-norm:

$$
a_{m}=\left[\sum_{f \in \widetilde{\mathscr{A}_{m}}}\left(\gamma_{m}(f)|Z(f)|\right)^{p}\right]^{1 / p}
$$

where $p$ is a positive number typically chosen relatively large to approximate the maximum. In this study, $p=8 . \widetilde{\mathscr{A}}_{m}$ is an extension of the domain $\mathscr{A}_{m}$ :

$$
\widetilde{\mathscr{A}_{m}}=\left\{f\left|\phi_{1} \geq\right| \phi(f)+2 \pi(m-1) \mid\right\}
$$

where $\phi_{0} \leq \phi_{1} \leq \pi$ to avoid any overlap between the $m^{\text {th }}$ resonance and other resonances. Finally $\gamma_{m}(f)$ is a weight function defined as:

$$
\left\{\begin{array}{ll}
\gamma_{m}(f)=\frac{1}{2}\left(1+\cos \left(\frac{\phi(f)-\phi_{0}}{\phi_{1}-\phi_{0}} \pi\right)\right), & \text { if } \phi_{0} \leq|\phi(f)+2 \pi(m-1)| \leq \phi_{1} \\
\gamma_{m}(f)=1 & \text { if }|\phi(f)+2 \pi(m-1)| \leq \phi_{0} \\
\gamma_{m}(f)=0 & \text { if }|\phi(f)+2 \pi(m-1)| \geq \phi_{1}
\end{array} .\right.
$$

This weight function is introduced to avoid discontinuities of numerical nature at the boundary of $\mathscr{A}_{m}$. The value assigned to these thresholds appears not to be critical for optimization as long as the inequality $\phi_{0} \leq \phi_{1} \leq \pi$ is respected. Based on numerical experiments, the thresholds $\phi_{0}$ and $\phi_{1}$ are set to $\pi / 4$ and $0.9 \pi$ respectively.

\section{Optimization problem}

\subsection{General formulation}

When playing a woodwind instrument several fingerings are used. Each fingering is associated with a specific input impedance, which changes as a function of the states of the tone holes and the register hole (open or closed). From a modeling point of view, the two states of a hole translate into different transfer matrices (Sec. 2). 
In this study, the purpose of the optimization is to adjust, for all fingerings, the resonance frequencies as well as the ratio of the magnitudes of the corresponding peaks.

Specifically, consider the $m^{\text {th }}$ resonance frequency for the $i^{\text {th }}$ fingering, and a corresponding target (desired) frequency $f_{m, i}^{\odot}$. Using the phase definition developed in Section 3.1, one can define the "error" between the actual and target frequency as follows:

$$
\Phi_{m, i}(\mathbf{x})=\left(\frac{\phi\left(f_{m, i}^{\odot}\right)}{2 \pi}+(m-1)\right)^{2} .
$$

Similarly, to enforce the ratio between two peak amplitudes $a_{2, i}$ and $a_{1, i}$, the residual $A_{i}$ reads:

$$
A_{i}(\mathbf{x})=\left(\frac{a_{2, i} / a_{1, i}-r_{i}^{\odot}}{r_{i}^{\odot}}\right)^{2},
$$

with $r_{i}^{\odot}$ the target ratio for the $i^{\text {th }}$ fingering.

The general formulation of the optimization problem we wish to solve is:

$$
\begin{array}{cl}
\min _{\mathbf{x}} & P(\mathbf{x}) \\
\text { s.t. } & \mathbf{h}_{\text {geo }}(\mathbf{x}) \leq 0 \\
& \mathbf{g}(\mathbf{x}) \leq 0 \\
& \mathbf{x}_{\min } \leq \mathbf{x} \leq \mathbf{x}_{\max }
\end{array}
$$

where $P$ and $\mathbf{g}$ are the cost function and constraints involving a combination of $\Phi_{m, i}$ and $A_{i}$. $\mathbf{h}_{g e o}$ are geometric constraints related to the spatial positioning and hole dimensions. The vector of design variables is scaled so that and $\mathbf{x}_{\min }=0$ and $\mathbf{x}_{\max }=1$.

\subsection{Numerical aspects}

The phase computation $\phi(f)$ in Equation (12) requires a differentiation and an integration. While the differentiation is computed through finite differences, the integration is carried out using the rectangle method. Both operations are sensitive to the frequency step selected. A constant step of $1 \mathrm{~Hz}$ over the frequency range appears to be a good compromise between accuracy and computational time. For the cost function $\Phi$, it is necessary to evaluate the corresponding phase at the prescribed target frequencies (Eq. 17). To avoid an interpolation, the target frequencies are added to the set of frequencies used for the impedance computation.

In this study, optimizations are carried out using Sequential Quadratic Programming (SQP). This is a wellknown gradient-based algorithm for nonlinear optimization problems ([25], Chap.18). It is implemented in MATLAB through the fmincon function. SQP solves a nonlinear optimization problem through a succession of convex subproblems based on a quadratic approximation of the cost function and a linearization of the constraints. The Hessian is not exactly computed but it is approximated and iteratively updated using the gradient and the function values of the cost function. As any gradient-based method, SQP is a local optimization method and might converge to a local minimum in the neighborhood of the initialization, which must therefore be chosen carefully. At convergence, the Karush-Kuhn-Tucker (KKT) optimality conditions must be satisfied ([25], Chap.12). The KKT conditions are first-order and are therefore only necessary. In this study, the tolerance for the first order optimality condition is set to $10^{-6}$ while the tolerances on a relative change in design variables and cost function are set to $10^{-10}$ and $10^{-6}$ respectively. The gradients are estimated by centered finite differences with a step size of $10^{-7}$. This value was found after a systematic numerical investigation and showed to provide a good compromise between accuracy of the finite differences and numerical noise.

\section{Results for an illustrative instrument}

The instrument treated in this study is a fictive instrument including geometric constraints which could correspond to ergonomic constraints for a real instrument. The objective is to design a two-register clarinet-like instrument (a second register one octave and a fifth higher than the first register) which can be played without keypads and without forked-fingerings (logical instrument [16]) on a range similar to a traditional clarinet (from $D_{3}$ to $G_{4} \sharp$ for the sounding pitch of the first register of a $B b$ clarinet). Following this short design specification, the first register range of one octave and a half must be covered by, at most, nine tone holes (plus one register hole), which implies at most five notes per octave. A pentatonic scale is therefore chosen: a C-major pentatonic 
scale from $\mathrm{D}$ for the first register, giving a G-major pentatonic scale for the second register (Table 3). The range of this instrument is similar to the one of a traditional $B$ b clarinet $\left(D_{3}\right.$ to $A_{4}$ for the first register).

This results section describes the design variables (Sec. 5.1), the geometric constraints (Sec. 5.2), the targeted impedance characteristics (Sec. 5.3), as well as the method used to obtain the starting design for the optimization (Sec. 5.4). Three illustrative design optimization problems, all subjected to geometric constraints, are investigated:

1. the tuning of the first register frequencies only (Sec. 5.5)

2. the tuning of the first and second register frequencies (Sec. 5.6)

3. the ratio of magnitudes of the first two resonance peaks are optimized under the tuning constraints of the two registers (Sec. 5.7)

\subsection{Design variables}

The geometry of this instrument must be composed of at least eleven elements: the pipe entrance $(n=1)$, one register hole $(n=2)$, eight tone holes $(n \in[3,10])$ and the pipe end $(n=11)$. The main pipe radius at the entrance is fixed at $r_{\text {pipe }}(1)=7.45 \mathrm{~mm}$, which is the typical output radius of a $B$ b clarinet mouthpiece.

\begin{tabular}{l|c|l|} 
Elements & Type & Design variables \\
\hline$n=1$ & entrance & $0:$ all fixed \\
\hline$n=2$ & register hole & $4: r_{\text {pipe }}, l, r_{\text {hole }}, h_{\text {hole }}$ \\
\hline$n \in \llbracket 3,10 \rrbracket$ & tone holes & $4: r_{\text {pipe }}, l, r_{\text {hole }}, h_{\text {hole }}$ \\
\hline$n=11$ & final term. & $2: r_{\text {pipe }}$ and $l$ \\
\hline
\end{tabular}

Table 1: Geometry elements

The design variables are (Fig. 1):

- for each hole: the hole radius $r_{p i p e}(n)$, the chimney height $h_{\text {hole }}(n)$, the tone hole spacing $l(n)$, and the main pipe radius $r_{\text {pipe }}(n)$ at the tone hole location.

- the main pipe radius at the end $r_{\text {pipe }}(11)$ and its distance to the last hole $l(11)$

for a total of $N_{x}=38$ design variables (Table 1 ).

\subsection{Variable ranges and geometric constraints}

From a manufacturing point of view, there are three types of geometric constraints. Some are physical: a side hole cannot be wider than the main pipe; some are linked to the manufacturing process: if the main pipe is drilled with a reamer, only monotonic conicity can be built; and some are ergonomic: if the holes are blocked directly by the fingers, the hole radii and their spacing are limited by the dimensions of the human hand. Mathematically, they are organized in this study according to two types: side constraints (restricted authorized range) and linear inequality constraints.

\begin{tabular}{c|c|r|r|} 
Param. & Elements & Min. & Max. \\
\hline \hline$r_{\text {pipe }}$ & $\forall n$ & $5 \mathrm{~mm}$ & $15 \mathrm{~mm}$ \\
\hline \hline$h_{\text {hole }}$ & $\forall n$ & $3 \mathrm{~mm}$ & $20 \mathrm{~mm}$ \\
\hline \hline$r_{\text {hole }}$ & $n=2$ & $1 \mathrm{~mm}$ & $7.5 \mathrm{~mm}$ \\
\hline$r_{\text {hole }}$ & $n \in\{3,4,5,7,8,9\}$ & $2 \mathrm{~mm}$ & $6 \mathrm{~mm}$ \\
\hline$r_{\text {hole }}$ & $n \in\{6,10\}$ & $2 \mathrm{~mm}$ & $5 \mathrm{~mm}$ \\
\hline \hline$l$ & $n \in\{2,3,11\}$ & $1 \mathrm{~mm}$ & $150 \mathrm{~mm}$ \\
\hline$l$ & $n \in\{4,5,6,8,9,10\}$ & $10 \mathrm{~mm}$ & $30 \mathrm{~mm}$ \\
\hline$l$ & $n=7$ & $10 \mathrm{~mm}$ & $150 \mathrm{~mm}$ \\
\hline \hline
\end{tabular}

Table 2: Boundaries of the variables of design

The side constraints are provided in Table 2. The range of the main pipe radius $r_{p i p e}$ is chosen to prevent excessively steep flares or strangles. The range of the hole radii are selected to be smaller than the dimensions 
of regular finger tips (larger for the thumb $(n=2)$ and thinner for the little fingers $(n \in\{6,10\})$ ), but wide enough to avoid nonlinear effects which could be important in small holes [26]. Except for the thumb, the spacing $l(n)$ between successive holes should be between $10 \mathrm{~mm}$ and $30 \mathrm{~mm}$ for the fingers of one given hand. The other distances including that between the hands $(n \in\{2,3,11\})$ can vary freely, which is why a large upper bound $(150 \mathrm{~mm})$ was chosen.

In addition to the side constraints, linear relations between parameters are imposed. All tone hole radii must be smaller than the main pipe radius with a margin of $1 \mathrm{~mm}$ :

$$
r_{\text {hole }}(n)-r_{\text {pipe }}(n) \leq-1 \mathrm{~mm} \quad \text { for } n \in \llbracket 2,10 \rrbracket \text {. }
$$

The relation

$$
r_{\text {hole }}(n-1)+r_{\text {hole }}(n)-l(n) \leq-4 \mathrm{~mm} \quad \text { for } n \in \llbracket 4,10 \rrbracket,
$$

avoids the overlapping of tone holes and ensures that the tone holes can be covered correctly by the fingers, by guaranteeing a minimal distance of $4 \mathrm{~mm}$ between tone holes edges, excluding the register hole. In actual clarinets, the register tone hole chimney sticks out into the main pipe. Therefore, the register hole height is limited by the bore diameter:

$$
h_{\text {hole }}(2)-2 r_{\text {pipe }}(2) \leq-1 \mathrm{~mm},
$$

here again a margin of $1 \mathrm{~mm}$ is imposed. Finally, due to the conical sections, a condition on the chimney height must be added to ensure that the top of the chimneys protrudes through the main pipe. The following relations are imposed for all holes $(n \in \llbracket 2,10 \rrbracket)$ (see Fig. 1)

$$
\left\{\begin{array}{l}
h_{\text {hole }}(n)+r_{\text {pipe }}(n) \geq r_{\text {pipe }}(n-1) \\
h_{\text {hole }}(n)+r_{\text {pipe }}(n) \geq r_{\text {pipe }}(n+1)
\end{array},\right.
$$

which imposes that the top of a chimney is more distant to the main axis than the main pipe at the surrounding holes.

Note that in this work, the constraints linked to the use of specific tools during the manufacturing process are not considered. To simplify the optimization problem presentation, all the linear relations between the variables are grouped under one inequality constraint $\mathbf{h}_{\text {geo }}(\mathbf{x}) \leq 0$.

\section{$5.3 \quad$ Target values}

The choice of target values is a crucial aspect in the design of a new instrument. Although this work is a collaboration with the manufacturer Buffet Crampon, the strategy used to fix these values is not provided for confidentiality reasons. However, the aim of this study is to present the optimization process to design a resonator with the desired acoustic characteristics under geometric constraints. It is not to design an actual instrument which plays in tune. For these reasons, target characteristics are chosen somewhat arbitrarily, while being consistent with the behavior of a clarinet-like instrument.

\begin{tabular}{|c|c|c|c|c|c|c|c|c|c|}
\hline Fingering index $i$ & 1 & 2 & 3 & 4 & 5 & 6 & 7 & 8 & 9 \\
\hline Notes & $D_{3}$ & $E_{3}$ & $G_{3}$ & $A_{3}$ & $C_{4}$ & $D_{4}$ & $E_{4}$ & $G_{4}$ & $A_{4}$ \\
\hline Open tone holes & 0 & 1 & 2 & 3 & 4 & 5 & 6 & 7 & 8 \\
\hline$f_{1, i}^{\odot}(\mathrm{Hz})$ & 147 & 165 & 196 & 220 & 262 & 294 & 330 & 392 & 440 \\
\hline$r_{i}^{\odot}$ & 0.5 & 0.5 & 0.5 & 0.5 & 0.5 & 0.5 & 0.5 & 0.5 & 0.5 \\
\hline
\end{tabular}

a) First register: register hole closed

\begin{tabular}{|c|c|c|c|c|c|c|c|c|c|}
\hline Fingering index $i$ & 10 & 11 & 12 & 13 & 14 & 15 & 16 & 17 & 18 \\
\hline Notes & $A_{4}$ & $B_{4}$ & $D_{5}$ & $E_{5}$ & $G_{5}$ & $A_{5}$ & $B_{5}$ & $D_{6}$ & $E_{6}$ \\
\hline Open tone holes & 0 & 1 & 2 & 3 & 4 & 5 & 6 & 7 & 8 \\
\hline$f_{2, i}^{\odot}(\mathrm{Hz})$ & 440 & 494 & 587 & 659 & 784 & 880 & 988 & 1175 & 1319 \\
\hline$r_{i}^{\odot}$ & 2 & 1.89 & 1.74 & 1.63 & 1.47 & 1.37 & 1.26 & 1.11 & 1 \\
\hline
\end{tabular}

b) Second register: register hole open

Table 3: For each fingering: the index $i$, the corresponding note, the number of open tone holes, the target frequency for the first resonance $f_{1, i}^{\odot}$ for the first register (Tab. $3 a$ ) and the second resonance $f_{2, i}^{\odot}$ for the second register (Tab. 3 b) and the corresponding resonance amplitude ratios $r_{i}^{\odot}$.

In the case of the first register (register hole closed), the target frequencies $f_{1, i}^{\odot}$ for the various fingerings follow an equal temperament with $A_{4}=440 \mathrm{~Hz}$. In this study, the effect of the harmonicity on the sound is neglected 
and the second resonance frequencies are ignored for the first register. Similarly, the first resonances are ignored in the case of the second register (register hole open) and the target frequencies for the second resonances also follow an equal temperament.

The aim of a register hole is to decrease the amplitude of the first resonance peak. For this reason, a third quantity is introduced: the ratio of the magnitude of the two first resonances $a_{2} / a_{1}$, as suggested by Guilloteau [20, 17]. To facilitate the sound production in a clarinet-like instrument, target values for the amplitude ratios are introduced for both registers so that the played peak has the higher magnitude. For the first register $(i \in[1,9])$, the first peak must be higher. The target value of this ratio is set to $r_{i}^{\odot}=0.5$, which is a typical value for a cylinder. For the second register, reaching the second resonance is facilitated if the ratio is larger than 1. Following the study by Guilloteau [20,17], it has been observed experimentally that $r_{i}$ decreases linearly with respect to the pitch for one register hole. In this study, an arbitrary choice of amplitude ratios varying linearly from 2 at the bottom of the second register to 1 at its top has been made. Frequency and amplitude ratio targets are summarized in Table 3.

\subsection{Low frequency approximation as a starting design}

As proposed by Noreland [16], the location of the tone holes can be estimated analytically through a low frequency approximation. The corresponding geometry can then be used as a starting point of the optimization algorithm. Based on the low frequency approximation, the geometric elements are modeled by inertances, which can be interpreted as length corrections from the entrance cross-section $\left(\pi r_{p i p e}(1)^{2}\right)$ leading to an effective location $P_{\text {eff }}(n)$ of each hole including:

- the effective length of the tone hole, including: the chimney height $\left(h_{\text {hole }}\right)$, the matching volume, the "inner length" correction and the radiation length corrections [21],

- the length correction due to the downstream part of the instrument [1, Chap.7.7.5.3, p.375]

- the length correction induced by the volume added by the closed chimneys located upstream [27].

Due to the rough approximations, if the effective location of the holes are fixed to the quarter of the target wavelength, the resonance frequencies of the computed impedance have an average offset of -20 cents. To account for the bias, it is included in the target locations: $P_{\text {eff }}(n)=c /\left(4 f_{1} \times 2^{-20 / 1200}\right)$, where $c$ is the sound velocity. If possible, the register hole is positioned at the third of the mean of the tone holes effective locations with the largest chimney height and the smallest radius so as to maximize its acoustic mass.

The design variables are modified following this low-frequency approximation by respecting the side constraints provided in Sec. 5.2. The distances $l$ are modified first, then the chimney heights $h_{\text {hole }}$ and finally the hole radii $r_{\text {hole. }}$. The main pipe radii are set to an arbitrary value equal to the pipe entrance radius: $r_{\text {pipe }}(n)=r_{\text {pipe }}(1)=7.45 \mathrm{~mm}$ for all $n$.

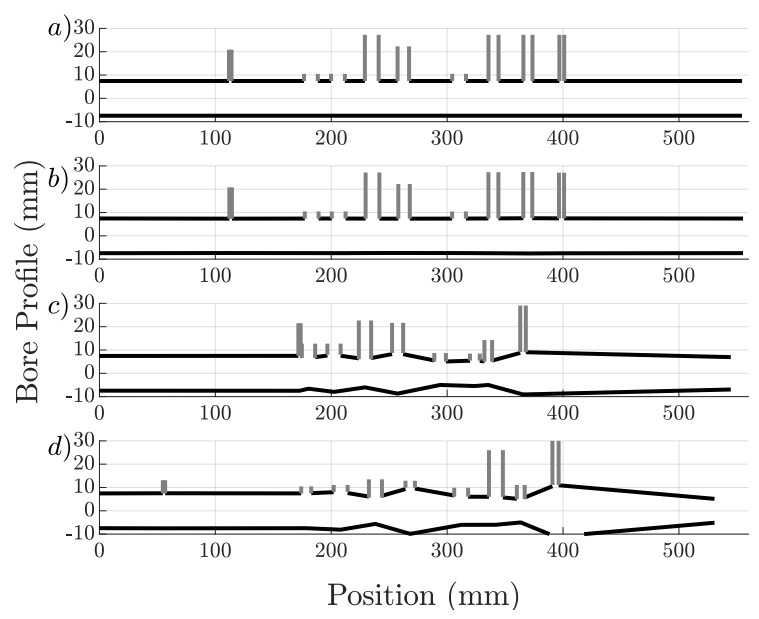

Figure 6: Comparison of geometries: (a) initial geometry (Sec. 5.4), the geometries obtained after the optimization of: $(b)$ the first resonance frequency $F$ (Sec. 5.5); $(c)$ the two registers tuning $F+H$ (Sec. 5.6); $(d)$ the ratio of magnitude of the two first resonances $A$ under the constraint on the resonance frequencies (Sec. 5.7). 
The geometry obtained for targets of Table 3.a by this low-frequency initialization process is depicted in Figure 6.a. The corresponding tuning for this initial step is represented on Figure 7. The deviation between the first resonance frequencies and the target frequencies is within \pm 10 cents except for the second fingering $F \sharp_{3}$. This is because all the modifiable parameters of the last hole $(n=10)$ reach their bounds (the distance $l(10)$, the chimney height $h_{\text {hole }}(10)$, and the hole radius $\left.r_{\text {hole }}(10)\right)$. Due to the constraints, it should not be surprising that the process presented does not allow a satisfying tuning of the analytically designed geometry for all fingerings. However, this geometry provides a good starting point for the optimization described in the subsequent paragraph.

\subsection{Problem formulation 1: first register tuning}

The first optimization problem deals with the tuning of the first register (9 fingerings)(Table 3.a). Based on Sections 3.1 and 4.1, the cost function for 9 fingerings reads:

$$
F(\mathbf{x})=\frac{1}{9} \sum_{i=1}^{i=9} \Phi_{1, i}=\frac{1}{9} \sum_{i=1}^{i=9}\left(\frac{\phi\left(f_{1, i}^{\odot}\right)}{2 \pi}\right)^{2}
$$

In this problem, only geometric constraints (variables ranges and their linear relations, Sec. 5.2) are considered, thus leading to the following optimization problem:

$$
\begin{array}{cl}
\min _{\mathbf{x}} & F(\mathbf{x}) \\
\text { subject to } & \mathbf{h}_{\text {geo }}(\mathbf{x}) \leq 0 \\
& 0 \leq \mathbf{x} \leq 1
\end{array}
$$

The cost function $F$ only depends on the phase $\phi$ computed at the first register target frequencies $f_{1, i}^{\odot}$ with impedances computed for the 9 first fingerings (Tab. 3.a). It is noteworthy that it is not necessary to compute impedances for frequencies higher than the maximum target $f_{1, i}^{\odot}$. Since the highest frequency of interest is $440 \mathrm{~Hz}$ (Tab. 3.a), the frequency range is fixed to $[0.1,480] \mathrm{Hz}$.

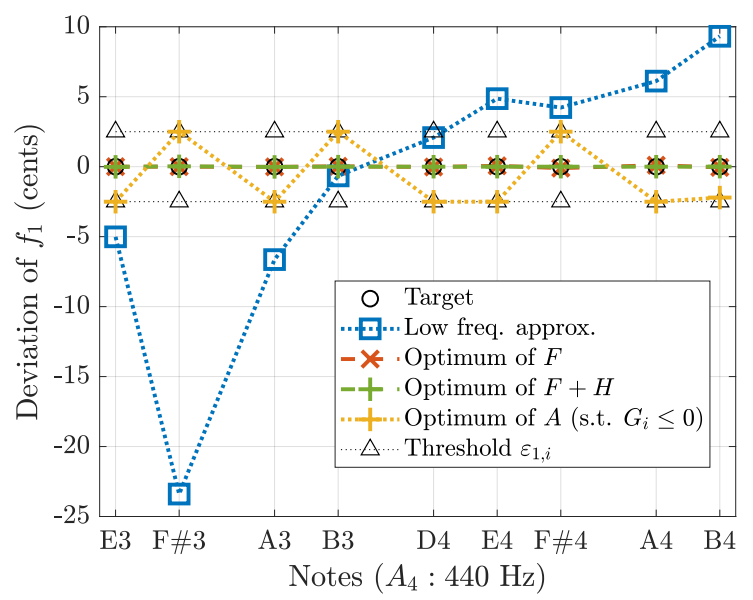

Figure 7: Deviation in cents of the first resonance frequencies from the target value for the first register (9 first fingerings) for the starting design (Sec. 5.4) and for the geometries obtained after optimization of Problem 1 (Sec. 5.5), Problem 2 (see Sec. 5.6) and Problem 3 with the corresponding $\varepsilon_{1, i}$ tuning bounds (see Sec. 5.7).

The SQP algorithm is initialized with the geometry obtained using the low frequency approximation described in Sec. 5.4. The algorithm converges in 35 iterations computed in less than 1 minute $(<1.7$ sec/iteration $)$ on a standard personal computer ( 4 cores, $16 \mathrm{~Gb}$ of RAM). The cost function reaches $F \approx 10^{-9}$ corresponding to deviations within 0.1 cents between the first resonance frequencies $f_{1, i}$ and their targets $f_{1, i}^{\odot}$ (Fig. 7) (the resonance frequencies $f_{1, i}$ are associated with the zeros of the phase $\phi$ estimated trough a linear interpolation around the zero-crossing). The optimal geometry is depicted in Figure 6.b. The design is very close to the initial geometry (Fig. 6.a) with design variables modified by less than $1.5 \%$ except for the last tone hole radius $\left(r_{\text {hole }}(10)\right)$, which is modified by about $6 \%$. 


\subsection{Problem formulation 2: two registers tuning}

In the second optimization problem, the simultaneous tuning of the two first registers is considered. The cost function for the 9 fingerings of the second register (Table 3.b) reads:

$$
H(\mathbf{x})=\frac{1}{9} \sum_{i=10}^{i=18} \Phi_{2, i}=\frac{1}{9} \sum_{i=10}^{i=18}\left(\frac{\phi\left(f_{2, i}^{\odot}\right)}{2 \pi}+1\right)^{2} .
$$

Accounting for the two first registers, the optimization problem with geometric constraints is:

$$
\begin{array}{cl}
\min _{\mathbf{x}} & (F(\mathbf{x})+H(\mathbf{x})) \\
\text { s. t. } & \mathbf{h}_{\text {geo }}(\mathbf{x}) \leq 0 \\
& 0 \leq \mathbf{x} \leq 1
\end{array}
$$

For this problem, the highest frequency of interest is $1319 \mathrm{~Hz}$ (Tab. 3.b) and the frequency range chosen is $[0.1,1450] \mathrm{Hz}$.

a)

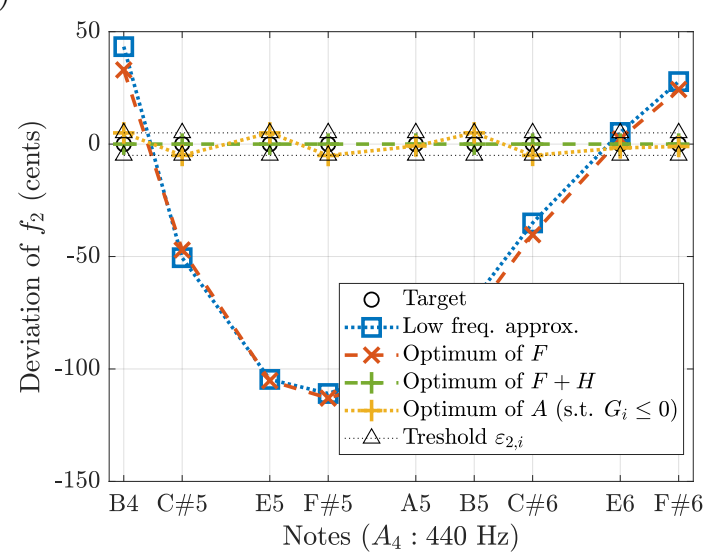

b)

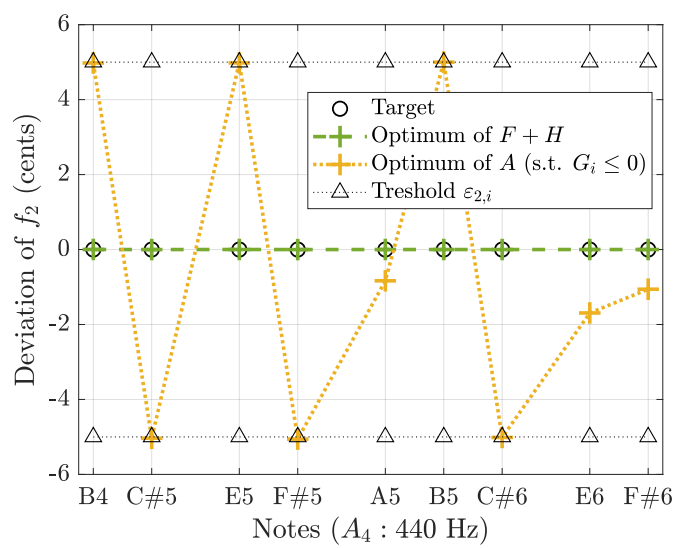

Figure 8: Deviation in cents of the second resonance frequencies from the target values for the 9 second register fingerings. a) for the four geometries of Fig 6: the starting design (Sec. 5.4) and the solutions of the three problems (Sec. 5.5, 5.6 and 5.7); b) a zoom within \pm 5 cents for the geometries for which the second resonance frequency has been optimized: Problems 2 (Sec. 5.6) and 3 (Sec. 5.7). The tuning bounds $\varepsilon_{2, i}$ are presented in Sec. 5.7.

Once again, the optimization algorithm is initialized with the geometries obtained in Section 5.4. The algorithm converges in 451 iterations (about 1 hour on a personal computer). The computation time per iteration (about $8 \mathrm{sec}$ /iteration) is longer than for the first problem (Sec. 5.5) because the frequency range used is wider and it is necessary to compute two times more impedances per iteration (18 fingerings against 9 for the first problem). The final cost values are $F \approx 10^{-11}$ and $H \approx 10^{-12}$, which corresponds to deviations within 0.025 cents for the first register and 0.002 cents for the second one (Fig. 7, Fig. 8).

The optimal geometry in depicted in Figure 6.c. This time, due to the addition of the cost function $H$, the algorithm converges to a geometry substantially different from the starting design (Fig. 6). The abrupt changes in the profile of the optimal is a very different result from other studies Noreland et al. [16], where smoother solutions were reached when more fingerings were considered. This difference stems from the geometric constraints which are active at the optimum. It is also noteworthy that the optimum reached is better both for $H$ and $F$ than the optimum obtained in the first problem where only $F$ was considered (Sec. 5.5): here $F \approx 10^{-11}$ vs. $F \approx 10^{-9}$ for the first problem. This means that a local optimum was reached in the first problem which was further evidenced by the fact that the optimal solution was very close to the starting design.

\subsection{Problem 3: tuning of the two first register accounting for impedance ampli- tude ratios}

In order to have a playable second register, it is necessary to adjust the relative magnitude of the two first peaks [17]. As introduced in Sec. 4.1, we wish to minimize the difference between the ratio of the two first 
impedance peaks and a target ratio. For all fingerings of the two first registers, this difference is quantified using the following quantity:

$$
A(\mathbf{x})=\frac{1}{18} \sum_{i=1}^{i=18} A_{i}=\frac{1}{18} \sum_{i=1}^{i=18}\left(\frac{a_{2, i} / a_{1, i}-r_{i}^{\odot}}{r_{i}^{\odot}}\right)^{2}
$$

As a first step, one could consider the following optimization problem with three residuals $(F, H$, and $A)$ to minimize:

$$
\begin{array}{cl}
\min _{\mathbf{x}} & (F(\mathbf{x})+H(\mathbf{x})+A(\mathbf{x})) \\
\text { s. t. } & \mathbf{h}_{\text {geo }}(\mathbf{x}) \leq 0 \\
& 0 \leq \mathbf{x} \leq 1
\end{array}
$$

However, the local minimum reached using the starting geometry described in Sec. 5.4 is not satisfying. Indeed, at the end of the optimization, $A$ and $F$ are approximately $10^{-3}$ and $H \approx 10^{-4}$. This translates into deviations larger than 100 cents for some of the first register fingerings and 40 cents for the second register, which are not acceptable deviations. However, the ratios of magnitudes are within $\pm 10 \%$ of their targets. Although the ratio residuals are satisfactory results from an optimization standpoint (local minimum), this aspect is not critical for musical instruments. Experiments from different starting geometries suggest that it is impossible to simultaneously reach the targets of the three cost functions.

To solve this problem, one possibility is to convert the cost functions $F$ and $H$ into nonlinear constraints which impose an allowable deviation between the resonance frequencies and their targets. For the $i^{\text {th }}$ fingering, the maximum deviation $\varepsilon_{m, i}$ between the $m^{t h}$ resonance frequency $f_{m, i}$ and the target $f_{m, i}^{\odot}$ is based on the definition of a cent:

$$
\varepsilon_{m, i} \geq 1200 \log _{2}\left(\frac{f_{m, i}}{f_{m, i}^{\odot}}\right) .
$$

This study used an allowable deviation of 2.5 cents for the first register $(i \in[1,9])$ and 5 cents for the second register $(i \in[10,18])$.

To convert $F$ and $H$ into inequality constraints, one must find the corresponding allowable phases. The maximum allowed deviation in terms of the angle $\phi$ of the reflection function at the target frequency $f_{m, i}^{\odot}$ is expressed as $\zeta_{m, i}$. Assuming $f_{n, i}$ is close to $f_{m, i}^{\odot}$, a first-order Taylor development about a target frequency gives:

$$
\phi\left(f_{n, i}^{\odot}\right)=\phi\left(f_{n, i}\right)-f_{n, i}^{\odot}\left(\frac{f_{n, i}}{f_{n, i}^{\odot}}-1\right) \frac{d \phi}{d f}\left(f_{n, i}^{\odot}\right) .
$$

This approximation remains valid in the region where the phase evolves linearly with respect to the frequency. This validity domain can be bounded, for instance, by a double resonance. Figure 3 b depicts an extreme case where the domain is \pm 15 cents around a resonance, which is wide enough for the purpose of this study. For the chosen allowable deviations $\left(\varepsilon_{m, i}=2.5\right.$ cents and $\varepsilon_{m, i}=5$ cents), the proposed linear approximation provides satisfactory results as illustrated in the following results (Fig. 7 and Fig. 8, b).

Using Eq. 30, Eq. 31 and the fact that $\phi\left(f_{m, i}\right)=-2 \pi(m-1)$ at resonance, the phase threshold $\zeta_{m, i}$ is therefore defined as:

$$
\zeta_{m, i}=(1-m)-\frac{1}{2 \pi} f_{m, i}^{\odot}\left(2^{\left(\varepsilon_{m, i} / 1200\right)}-1\right) \frac{d \phi}{d f}\left(f_{m, i}^{\odot}\right) .
$$

This threshold depends only on the target frequency considered and the derivative of the phase at this frequency. Numerically, this derivative is estimated from a spline interpolation around $\phi\left(f_{m, i}^{\odot}\right)$. It is now possible to construct nonlinear constraints $G_{i}$ which are negative when the frequency deviation between $f_{m, i}$ and $f_{m, i}^{\odot}$ satisfies the allowable deviation:

$$
G_{i}=\left\{\begin{array}{l}
\Phi_{1, i}-\left(\zeta_{1, i}\right)^{2} \text { for } i \in[1,9] \\
\Phi_{2, i}-\left(\zeta_{2, i}\right)^{2} \text { for } i \in[10,18]
\end{array}\right.
$$

The third optimization problem can therefore be recast as:

$$
\begin{array}{ll}
\min _{\mathbf{x}} & A(\mathbf{x}) \\
\text { s. t. } & \mathbf{h}_{\text {geo }}(\mathbf{x}) \leq 0 \\
& G_{i}(\mathbf{x}) \leq 0 \quad \text { for } i \in[1,18] \\
& 0 \leq \mathbf{x} \leq 1
\end{array}
$$




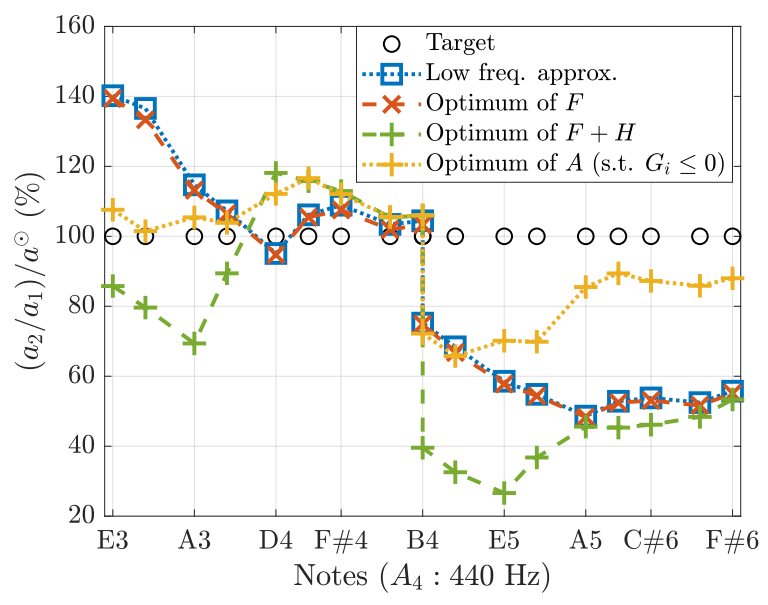

Figure 9: Ratio of peak amplitudes $a_{2} / a_{1}$ for the two first resonances for each fingering. Comparison to target ratios $a^{\odot}$ for the starting design (Sec. 5.4). Solutions of the three optimization problems (Sec. 5.5, 5.6, and 5.7).

Note that to correctly estimate the magnitude of the second peaks, the frequency range is extended to [0.1, 4000] Hz.

The optimization algorithm initialized with the low frequency approximation converges in 88 iterations (about $30 \mathrm{~min}$ on a personal computer). The longer computational time per iteration is expected due to the larger frequency range. All nonlinear constraints $G_{i}$ are satisfied, and 14 of the 18 are active: 8 on the first register (Fig. 7), and 6 on the second (Fig. 8.b). The deviation between the first resonance frequencies and their targets are within \pm 2.5 cents for the first register (Fig. 7) and within \pm 5 cents for the second resonance frequencies on the second register (Fig. 8.b). The optimum cost value is $A \approx 2.6 \cdot 10^{-2}$ which corresponds to amplitude ratio deviations to the targets within $20 \%$ for most of the fingerings (Fig. 9) with the exception of the lower second register where the maximum deviation is about 30\% (from B4 to G5). Compared to other solutions obtained, the improvement is especially pronounced in the lower part of the first register and the higher part of the second one (Fig. 9).

The optimal geometry (Fig. 6.d) is markedly different from the previous ones: the total length is shorter, the end is a convergent cone and the chimneys are overall shorter. Note that in this optimum geometry, the register hole is close to the entrance with a short chimney. This is a striking difference with the geometry obtained from the optimization of $F+H$ (Fig. 6.c) where the register hole was the closest possible to the first tone hole and had a long chimney. The influence of the register hole, and especially its chimney height, on the ratio of peak amplitudes had already been noted by Noreland [16] and Guilloteau [17].

\section{Local optima and influence of the initial geometry}

Since a gradient-based method is used, the optimizer might stop at a local optimum. To study the presence of local optima and therefore the dependence on the starting point, the three optimization problems (25), (27) and (34) are repeated using 20 starting geometries. The values of design parameters are selected randomly within their bounds (Table 2).

For problem 1, where only $F$ is minimized (Sec. 5.5), the optimizations converge to solutions with frequency deviations within 0.1 cents for all starting points. An average of 75 iterations was required with a mean optimal cost value of abound $10^{-12}$. The worst optimum was obtained using the low frequency approximation as a starting design with $\left(F \approx 10^{-9}\right)$. The fact that this could not be the best solutions was already discussed in Section 5.6 where Problem 2 reached a better solution for $F$ despite having to also minimize $H$.

The optimal solutions cover a wide variety of geometries (Fig. 10). This illustrates the fact that, the sole minimization of $F$ leads to many geometries with acceptable residuals. This gives the possibility for the manufacturer to choose between the available solutions or to complicate the problem by adding, for example, manufacturing constraints.

In Problem $2(F+H)$, a wide range of solutions is obtained. 15 out of 20 optimizations reached acceptable frequency deviations $\left(F+H<10^{-9}\right.$, corresponding to a deviation within 0.5 cents). However, 4 solutions converge to a cost function $F+H>10^{-6}$ with deviations of about 10 cents. In one case, $F+H \approx 10^{-3}$ corresponding to an unacceptable deviation between the resonance frequencies and their targets of about 500 cents 


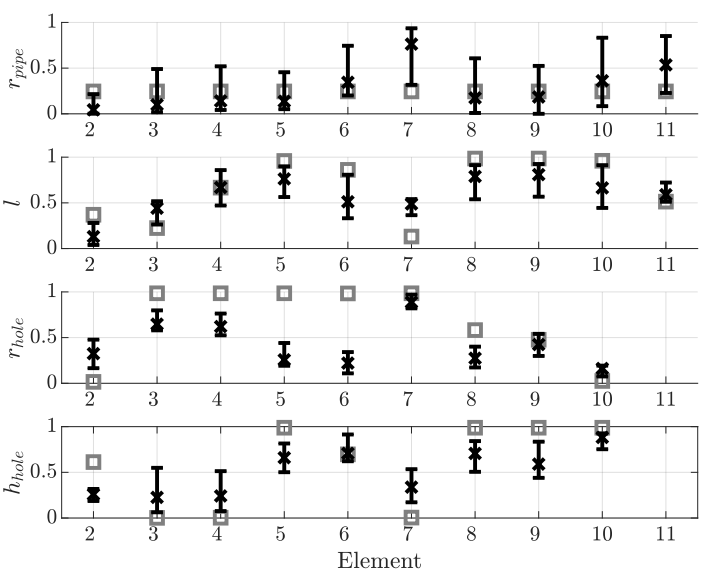

Figure 10: Variability of the normalized geometric parameters of the 20 geometries obtained for Problem 1 (Eq. 25, Sec. 5.5). The optimal values of $F$ range between $10^{-14}$ and $10^{-9}$. The boxplot provides the variable medians (crosses), the $90 \%$ interval bounds (horizontal bars), and the low frequency approximation geometry (gray squares).

for some fingerings!

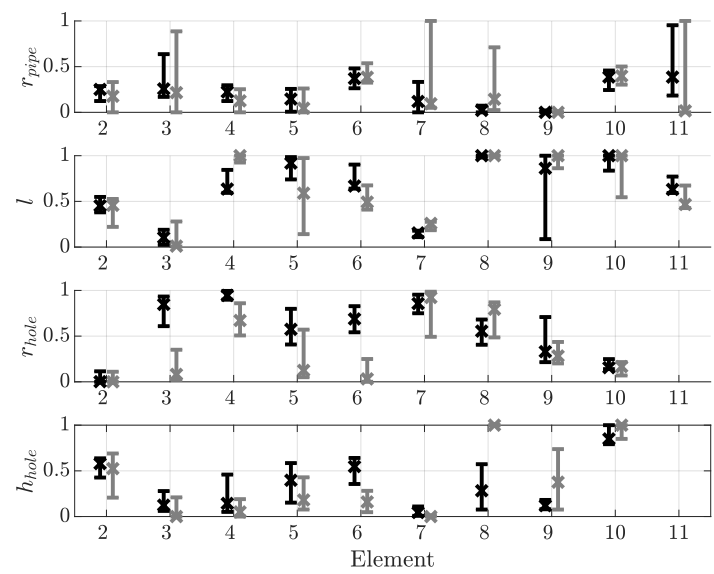

Figure 11: Variability of the normalized geometric parameters of the 20 geometries obtained for Problem 2 (Eq. 27, Sec. 5.6). The boxplot provides the variable medians (crosses), the $90 \%$ interval bounds (horizontal bars). 15 Cases with $F+H<10^{-9}$ (black) and 5 cases with $F+H>10^{-6}$ (gray).

The optimal dimensions obtained for the two main groups of optima $\left(F+H<10^{-9}\right.$ and $\left.F+H>10^{-6}\right)$ are depicted in Figure 11. In the first group $\left(F+H<10^{-9}\right)$ most geometries are similar, except for the end element radius $r_{\text {pipe }}(11)$ and the location of the last three elements trough the distance $l(9)$. These two variables acoustically compensate each other. The two groups of geometries differ essentially by the hole radii $r_{\text {hole }}(3,5,6)$ and the chimney height $h_{\text {hole }}(8)$. These two groups correspond to two regions of the design space, leading to two types solutions as a function of the starting geometry.

For the problem 3 ( $A$ s.t. $G_{i}$, Sec. 5.7), only four optimization out of 20 led to satisfactory results. The other cases did not converge or had a very poor convergence rate and were stopped. For the 4 converged cases, an average of 180 iterations was required (about 45 minutes of computational time). Two optimal geometries, including the one with a low frequency approximation starting design, are identical with $A \approx 2.6 \cdot 10^{-2}$. The two other cases reached an optimum of about $A \approx 5 \cdot 10^{-3}$.

The observed convergence failure for 16 cases can have several origins. Non-differentiability of the cost functions or constraints or a poor approximation of the gradients are typical issues. Although the theoretical 
characterization of the resonances (Sec. 3.2) ensures their differentiability, non-smoothness might be introduced through the numerical implementation. Indeed, the numerical integration is used to compute Equation (12) and is sensitive to the frequency step, thus inducing numerical errors and a poor estimation of the finite differencebased gradients. Investigation is needed to further improve the numerical integration. The optimization can also be markedly improved through the derivation of analytical gradients, which is beyond the scope of the proposed work.

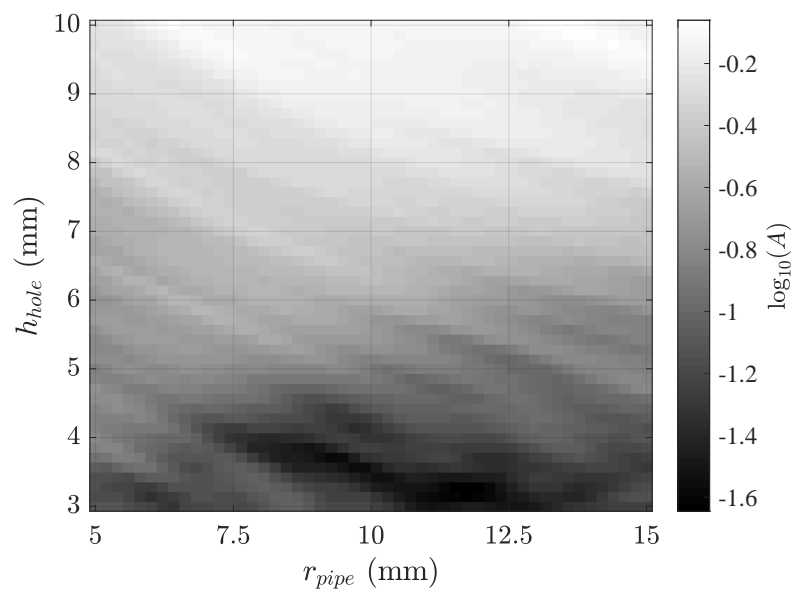

Figure 12: Map of the cost function $A$ in a two-dimensional space. All pipe radii are set equal to $r_{p i p e}$ and all chimney heights are set to $h_{\text {hole }}$. Other variables are fixed to their initial values. Several parallel valleys can be observed, potentially explaining the convergence difficulties encountered in Problem 3.

Another reason for the poor convergence stems from the topology of the 38 dimensional design space. As a graphical illustration in a lower dimensional representation, the cost function $A$ is depicted (Figure 12), in a reduced two-dimensional subspace created by linking the 9 chimney heights $h_{\text {hole }}(1)=h_{\text {hole }}(2)=\ldots=$ $h_{\text {hole }}(9)=h_{\text {hole }}$, and the 10 main pipe radii $r_{\text {pipe }}(1)=r_{\text {pipe }}(2)=\ldots=r_{\text {pipe }}(10)=r_{\text {pipe }}($ Tab. 1$)$. A series of valleys can be observed. This typically leads to slow convergence of the optimizer which is forced to progress slowly (often zig-zagging) with small steps. Depending on the convergence thresholds, the optimizer might stop before the KKT conditions are satisfied. Preliminary observations suggest that the presence of these valleys is inherent in the use of peak amplitudes. There is no specific study on the relation between the geometric parameters and the resonance amplitude. Specific investigations are needed to clarify this relation and eventually find a better way to include the resonance amplitude in the optimization process. With the definition used in this study, the initial geometry must be carefully selected to start the optimization in the neighborhood of a local minimum. This conclusion goes far beyond the specific case studied here.

\section{Conclusion}

This article deals with the design optimization of a woodwind instrument with geometric constraints. In order to obtain specific impedance characteristics (peak frequencies and peak amplitude ratios), new quantities are introduced. Specifically, resonances are identified based on an ad-hoc unwrapping of the phase of the reflection function. In addition, non-smoothness is eliminated through regularization. This makes it possible to use gradient-based optimization. The non-smoothness would be especially pronounced for complex frequency responses encountered in woodwind instruments with side holes. The geometry-constrained optimization implemented is carried out on an instrument with 38 variables thus demonstrating the potential of optimization to deliver new designs in relatively short computational time (around 1 hour).

Several aspects of this research need to be further developed. In particular, beside the problems presented in this articles, the inharmonicity between the first resonance frequencies for each fingering, or the radiation properties of the instrument could be considered. This could be done through a constrained or multiobjective formulation. In addition, this study has demonstrated that the computational optimal design of the woodwind instrument can be performed in a reasonable time, thus providing an opportunity to develop design-manufacturing (3D printing)-testing iterations, thus leading to instruments with excellent playability and characteristics. 


\section{Acknowledgments}

This work has been partly supported by the french Agence Nationale de la Recherche (ANR16-LCV2-0007-01 Liamfi project), in cooperation with Buffet Crampon.

\section{References}

[1] A. Chaigne and J. Kergomard, Acoustics of Musical Instruments. Modern Acoustics and Signal Processing, New York, NY: Springer New York, 2016.

[2] R. Caussé, J. Kergomard, and X. Lurton, "Input impedance of brass musical instruments - Comparison between experiment and numerical models," The Journal of the Acoustical Society of America, vol. 75, pp. 241-254, Jan. 1984.

[3] D. H. Keefe, "Acoustical wave propagation in cylindrical ducts: Transmission line parameter approximations for isothermal and nonisothermal boundary conditions," The Journal of the Acoustical Society of America, vol. 75, pp. 58-62, Jan. 1984.

[4] A. Lefebvre, Computational acoustic methods for the design of woodwind instruments. PhD thesis, McGill University, 2010.

[5] P. Eveno, J.-P. Dalmont, R. Caussé, and J. Gilbert, "Wave Propagation and Radiation in a Horn: Comparisons Between Models and Measurements," Acta Acustica united with Acustica, vol. 98, pp. 158-165, Jan. 2012.

[6] T. Hélie, T. Hézard, R. Mignot, and D. Matignon, "One-Dimensional Acoustic Models of Horns and Comparison with Measurements," Acta Acustica united with Acustica, vol. 99, pp. 960-974, Nov. 2013.

[7] J.-B. Doc, B. Lihoreau, S. Félix, and V. Pagneux, "Bremmer series for the multimodal sound propagation in inhomogeneous waveguides," Wave Motion, vol. 67, pp. 55-67, Dec. 2016.

[8] R. Tournemenne and J. Chabassier, "A comparison of a one-dimensional finite element method and the transfer matrix method for the computation of wind music instrument impedance," Acta Acustica united with Acustica, vol. 5, p. 838, 2019.

[9] Itemm, "Plateforme d'aide à la facture instrumentale," pafi.plateforme-lutherie.com, 2020.

[10] P. Eveno, L'impédance d'entrée pour l'aide à la facture des instruments de musique à vent : mesures, modèles et lien avec les fréquences de jeu. PhD Thesis, Université Pierre et Marie Curie - Paris VI, Dec. 2012.

[11] R. Tournemenne, J.-F. Petiot, B. Talgorn, J. Gilbert, and M. Kokkolaras, "Sound simulation-based design optimization of brass wind instruments," The Journal of the Acoustical Society of America, vol. 145, pp. 3795-3804, June 2019.

[12] W. Kausel, "Optimization of Brasswind Instruments and its Application in Bore Reconstruction," Journal of New Music Research, vol. 30, pp. 69-82, Mar. 2001.

[13] D. Noreland, "Gradient based optimisation of brass instruments," in Stockholm Music Acoustics Conference, (Stockholm, Sweden), 2003.

[14] A. C. P. Braden, M. J. Newton, and D. M. Campbell, "Trombone bore optimization based on input impedance targets," The Journal of the Acoustical Society of America, vol. 125, pp. 2404-2412, Apr. 2009.

[15] T. Colinot, P. Guillemain, J.-B. Doc, C. Vergez, and M. Jousserand, "Numerical Optimization of a Bicylindrical Resonator Impedance: Differences and Common Features Between a Saxophone Resonator and a Bicylindrical Resonator," Acta Acustica united with Acustica, vol. 105, pp. 1217-1227, Nov. 2019.

[16] D. Noreland, J. Kergomard, F. Laloë, C. Vergez, P. Guillemain, and A. Guilloteau, "The Logical Clarinet: Numerical Optimization of the Geometry of Woodwind Instruments," Acta Acustica united with Acustica, vol. 99, pp. 615-628, July 2013. 
[17] A. Guilloteau, P. Guillemain, J. Kergomard, and M. Jousserand, "On the Second Register's Playability of the Clarinet: Towards a Multicriteria Approach," in International Symposium on Musical Acoustics, (Montreal), p. 1, 2017.

[18] E. Moers and J. Kergomard, "On the Cutoff Frequency of Clarinet-Like Instruments. Geometrical versus Acoustical Regularity," Acta Acustica united with Acustica, vol. 97, pp. 984-996, Nov. 2011.

[19] E. Petersen, P. Guillemain, J. Kergomard, and T. Colinot, "The effect of the cutoff frequency on the sound production of a clarinet-like instrument," The Journal of the Acoustical Society of America, vol. 145, pp. 3784-3794, June 2019.

[20] A. Guilloteau, Conception d'une clarinette logique. PhD thesis, Aix-Marseille Université, Marseille, France, Sept. 2015.

[21] A. Lefebvre and G. P. Scavone, "Characterization of woodwind instrument toneholes with the finite element method," The Journal of the Acoustical Society of America, vol. 131, no. 4, pp. 3153-3163, 2012.

[22] J.-P. Dalmont, C. Nederveen, and N. Joly, "Radiation impedance of tubes with different flanges: numerical and experimental investigations," Journal of Sound and Vibration, vol. 244, pp. 505-534, July 2001.

[23] F. Silva, P. Guillemain, J. Kergomard, B. Mallaroni, and A. N. Norris, "Approximation formulae for the acoustic radiation impedance of a cylindrical pipe," Journal of Sound and Vibration, vol. 322, pp. 255-263, Apr. 2009.

[24] P. A. Taillard, F. Silva, P. Guillemain, and J. Kergomard, "Modal analysis of the input impedance of wind instruments. Application to the sound synthesis of a clarinet," Applied Acoustics, vol. 141, pp. 271-280, Dec. 2018.

[25] J. Nocedal and S. J. Wright, Numerical optimization. Springer series in operations research, New York: Springer, 2nd ed ed., 2006.

[26] J.-P. Dalmont, C. J. Nederveen, V. Dubos, S. Ollivier, V. Méserette, and E. te Sligte, "Experimental Determination of the Equivalent Circuit of an Open Side Hole: Linear and Non Linear Behaviour," Acta Acustica united with Acustica, vol. 88, pp. 567-575, July 2002.

[27] V. Debut, J. Kergomard, and F. Laloë, "Analysis and optimisation of the tuning of the twelfths for a clarinet resonator," Applied Acoustics, vol. 66, pp. 365-409, Apr. 2005. 